\title{
Adaptive Receive and Transmit Apodization for Synthetic Aperture Ultrasound Imaging
}

Holfort, Iben Kraglund; Austeng, Andreas; Synnevåg, Johan-Fredrik; Holm, Sverre; Gran, Fredrik; Jensen, Jørgen Arendt

Published in:

2009 IEEE International Ultrasonics Symposium (IUS)

Link to article, DOI:

10.1109/ULTSYM.2009.5442035

Publication date:

2009

Document Version

Publisher's PDF, also known as Version of record

Link back to DTU Orbit

Citation (APA):

Holfort, I. K., Austeng, A., Synnevåg, J-F., Holm, S., Gran, F., \& Jensen, J. A. (2009). Adaptive Receive and Transmit Apodization for Synthetic Aperture Ultrasound Imaging. In 2009 IEEE International Ultrasonics Symposium (IUS) IEEE. https://doi.org/10.1109/ULTSYM.2009.5442035

\section{General rights}

Copyright and moral rights for the publications made accessible in the public portal are retained by the authors and/or other copyright owners and it is a condition of accessing publications that users recognise and abide by the legal requirements associated with these rights.

- Users may download and print one copy of any publication from the public portal for the purpose of private study or research.

- You may not further distribute the material or use it for any profit-making activity or commercial gain

- You may freely distribute the URL identifying the publication in the public portal 


\title{
Adaptive Receive and Transmit Apodization for Synthetic Aperture Ultrasound Imaging
}

\author{
Iben Kraglund Holfort ${ }^{1}$, Andreas Austeng ${ }^{2}$, Johan-Fredrik Synnevåg ${ }^{2,3}$, Sverre Holm ${ }^{2}$, Fredrik Gran ${ }^{1,4}$ and \\ Jørgen Arendt Jensen ${ }^{1}$ \\ ${ }^{1}$ Department of Electrical Engineering, Technical University of Denmark, DK-2800 Kgs. Lyngby, Denmark \\ ${ }^{2}$ Department of Informatics, University of Oslo, P. O. Box 1080, N-0316 Oslo, Norway \\ ${ }^{3}$ Bergen Oilfield Services AS, Sandviksbodene 1E, NO-5035 Bergen, Norway \\ ${ }^{4}$ GN ReSound A/S, Lautrupbjerg 9, DK-2750 Ballerup, Denmark
}

\begin{abstract}
This paper suggests a framework for utilizing adaptive, data-dependent apodization weights on both the receiving and transmitting aperture for Synthetic Aperture (SA) ultrasound imaging. The suggested approach is based on the Minimum Variance (MV) beamformer and consists of two steps. A set of uniquely designed receive apodization weights are applied to pre-summed element data forming a set of adaptively weighted images; these are in SA literature conventionally referred to as low-resolution images. The adaptive transmit apodization is obtained by applying MV across the full set of single emission images before summation. The method is investigated using simulated SA ultrasound data obtained using Field II. Data of 13 point targets distributed at depths from $40 \mathrm{~mm}$ to $70 \mathrm{~mm}$, and a $5.5 \mathrm{MHz}, 64$-element linear array transducer have been used. The investigation has shown that the introduction of adaptive apodization weights on the transmitting aperture provides a main-lobe reduction (estimated at $-30 \mathrm{~dB}$ ) by a factor of 1.8 compared to the method using adaptive apodization weights on the receiving aperture only.
\end{abstract}

\section{INTRODUCTION}

Recently, the application of adaptive, data-dependent beamformers to the field of ultrasound imaging has become an area of increasing interest. These have provided significant results in terms of increased resolution and contrast compared to the conventional, delay-and-sum (DAS) beamformer [1]-[7].

Previously, the adaptive minimum variance (MV) beamformer has been applied to the receiving aperture of synthetic aperture ultrasound (SA) data [5]. This has provided single emission images with increased resolution. The set of single emission images are combined by a weighted sum to form the full SA image; these weights represent the transmit apodization. So-far only data-independent transmit apodization weights have been used.

This paper suggests a framework for utilizing adaptive, data-dependent apodization weights both on the receiving and transmitting aperture. The suggested approach is based on the MV beamformer and consists of two steps. A set of uniquely designed receive apodization weights are applied to pre-summed element data forming a set of adaptively weighted images; these are in SA literature conventionally referred to as low-resolution images. The adaptive transmit apodization is obtained by applying MV across the full set of single emission images before summation.
This paper investigates, whether the additional datadependent, adaptive apodization weights on the transmitting aperture provides an increased resolution compared to the data-independent transmitting apodization methods.

\section{Method}

The suggested method is based on a synthetic aperture ultrasound imaging framework, where a number of so-called lowresolution images are created from unfocused emissions; one image for each emission. These are combined by a weighted sum to form an image with an increased resolution. For more on SA see e.g. [8].

The principle of the proposed method is illustrated in Fig. 1. The method consists of two parts. First, adapted apodization weights are applied to the received data, one weight for each receive channel. This provides a set of adaptively weighted low-resolution images; one image for each emission. Subsequently, the chosen transmitting apodization weights

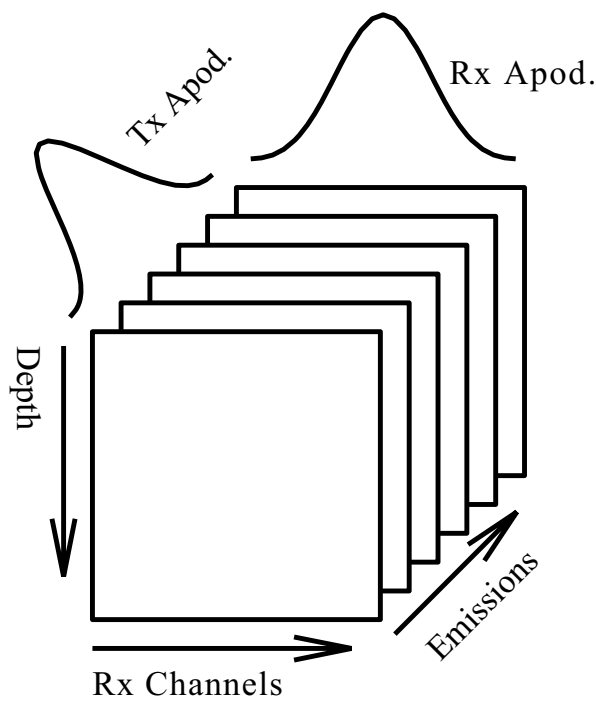

Fig. 1. Using a synthetic aperture (SA) imaging sequence a number of emissions are acquired. For each emission a data set is obtained, from each of these a single emission image created using a beamformer with the receiving $(\mathrm{Rx})$ apodization function. Weighting these images with the chosen transmitting (Tx) apodization function provides the combined SA image. 
are applied across these single emission images to form the combined SA image. This first part has previously been demonstrated in [5], where only data-independent apodization weights have been applied on the transmitting aperture.

The second part of the suggested method is to introduce data-dependent, adapted apodization weights on the transmitting aperture. In this way, the number of adapted weights and thereby the number of degrees of freedom are increased by a factor proportional to the number of SA emissions. Thus, the achievable resolution is assumed to increase significantly compared to both the data-independent transmitting apodization method and the conventional DAS method.

\section{A. Beamforming}

An ultrasound image is obtained using beamforming, where a specific set of time samples, $\Delta_{m, i}$, are chosen from the received sensor signals, $y_{m, i}(n)$. Which samples to choose is determined by the time-of-flight from the transmitting elements to the image point, $\vec{r}_{p}$, and back to the receiving elements. These samples are weighted and subsequently summed to form the beamformer output. To obtain an SA image, the set of so-called low-resolution images are furthermore weighed and summed to form the combined SA image, as illustrated in Fig. 1. Mathematically, an SA image is expressed by

$$
\begin{aligned}
b\left(\vec{r}_{p}\right) & =\sum_{i=0}^{I-1} a_{i}^{*}\left(\vec{r}_{p}\right) \sum_{m=0}^{M-1} w_{m, i}^{*}\left(\vec{r}_{p}\right) y_{m, i}\left(\Delta_{m, i}\left(\vec{r}_{p}\right)\right) \\
& =\sum_{i=0}^{I-1} a_{i}^{*}\left(\vec{r}_{p}\right) \ell_{i}\left(\vec{r}_{p}\right)
\end{aligned}
$$

where $a$ and $w$ denote the apodization weights on the transmitting and receiving apertures, respectively. The superscript, $\{\cdot\}^{*}$, denotes the complex conjugate. ${ }^{\mathrm{i}} I$ and $M$ are the number of emissions and receiving elements, respectively. And $\ell_{i}\left(\vec{r}_{p}\right)$ denotes the $i$ th low-resolution image. In matrix-vector notation, (2) becomes

$$
b\left(\vec{r}_{p}\right)=\mathbf{a}^{H}\left(\vec{r}_{p}\right) \boldsymbol{\ell}\left(\vec{r}_{p}\right)=\mathbf{a}^{H}\left(\vec{r}_{p}\right)\left[\begin{array}{c}
\mathbf{w}_{0}^{H} \mathbf{y}_{0}\left(\vec{r}_{p}\right) \\
\mathbf{w}_{1}^{H} \mathbf{y}_{1}\left(\vec{r}_{p}\right) \\
\vdots \\
\mathbf{w}_{I-1}^{H} \mathbf{y}_{I-1}\left(\vec{r}_{p}\right)
\end{array}\right],
$$

where a and $\left\{\mathbf{w}_{i}\right\}_{i=0}^{I-1}$ are vectors containing the apodization weights, $\ell$ contains the stacked low-resolution images, $\left\{\ell_{i}\left(\vec{r}_{p}\right)\right\}_{i=0}^{I-1}$, and the superscript, $\{\cdot\}^{H}$, denotes the Hermitian transpose.

\section{B. Adaptive Beamforming for both Receive and Transmit Apodization}

The adaptive apodization weights are found using the Minimum Variance (MV) beamformer, which was introduced by Capon in 1969 [9]. In the following, the apodization weight vector is denoted $\mathbf{u}$; it refers to and can be substituted for

\footnotetext{
${ }^{\mathrm{i} N o t e}$ that the complex conjugate operator is only introduced here to have a notation in consistency with the conventional matrix-vector notation. Using real apodization weights, the conjugation can be neglected.
}

the apodization weights on either the receiving aperture or the transmitting aperture.

The data-dependent MV beamformer uses apodization weights that are found from the measured wave field, as opposed to DAS, which uses pre-defined apodization weights. The MV beamformer finds a set of optimized weights that minimize the variance (or power) of the beamformer output, while passing the signal of interest without distortion. Thus, it passes the signal from the current receive focal point with unit gain. According to [9], the MV beamformer can mathematically be expressed as

$$
\begin{gathered}
\min _{\mathbf{u}} \mathbf{u}^{H} \mathbf{R u} \\
\text { subject to } \mathbf{u}^{H} \mathbf{e}=1
\end{gathered}
$$

where $\mathbf{e}$ is the so-called steering vector that defines the signal from the current receive focal point, $\vec{r}_{p}$, which should be passed with unit gain. $\mathbf{R}$ is the covariance matrix, described in Sec. II-B.1-II-B.2. The closed-form solution to (4) can be found using Lagrangian multiplier [10] theory as [9]

$$
\hat{\mathbf{u}}=\frac{\mathbf{R}^{-1} \mathbf{e}}{\mathbf{e}^{H} \mathbf{R}^{-1} \mathbf{e}},
$$

provided that $\mathbf{R}^{-1}$ exists.

The apodization weight vector, $\mathbf{u}$, refers to apodization on either the receiving aperture or the transmitting aperture. For optimization of the receive apodization weights for the $i$ th lowresolution image, $\mathbf{u}$ should be substituted by $\mathbf{w}_{i}$. For adapting the apodization weight on the transmitting aperture, $\mathbf{u}$ should be substituted by a.

Furthermore, the covariance matrix should be interchanged with either $\hat{\mathbf{R}}_{R x}$ or $\hat{\mathbf{R}}_{T x}$ according to which of the two apertures, Receive (Rx) or Transmit (Tx), is used. The estimation of the covariance matrices is described in the following.

1) Covariance Matrix Estimation for Receive Apodization: The covariance matrix for the receive apodization is estimated from the sensor signals, $\mathbf{y}_{i}\left(\vec{r}_{p}\right)$. For the $i$ th low-resolution image, the covariance matrix estimate is found as

$$
\hat{\mathbf{R}}_{R x}=\mathcal{E}\left\{\mathbf{y}_{i}\left(\vec{r}_{p}\right) \mathbf{y}_{i}\left(\vec{r}_{p}\right)^{H}\right\},
$$

where $\mathcal{E}\{\cdot\}$ denotes the expectation value.

2) Covariance Matrix Estimation for Transmit Apodization: For the transmit apodization, the adaptively weighted lowresolution images, $\ell\left(\vec{r}_{p}\right)$, are used to estimate the covariance matrix as

$$
\hat{\mathbf{R}}_{T x}=\mathcal{E}\left\{\boldsymbol{\ell}\left(\vec{r}_{p}\right) \boldsymbol{\ell}\left(\vec{r}_{p}\right)^{H}\right\}
$$

Note that $\hat{\mathbf{R}}_{R x}$ and $\hat{\mathbf{R}}_{T x}$ are both dependent on the image point, $\vec{r}_{p}$, however this dependency has been omitted to simplify notation.

3) Spatial Smoothing and Diagonal Loading: In real applications, the covariance matrix is unknown and must be estimated from data. Several realizations of data are required; these are obtained by smoothing the covariance matrix estimate spatially as suggested in [11]. Fig. 2 illustrates how the array is divided into $P$ overlapping subarrays of length $L$. For each subarray, a sub-covariance matrix is estimated. The 


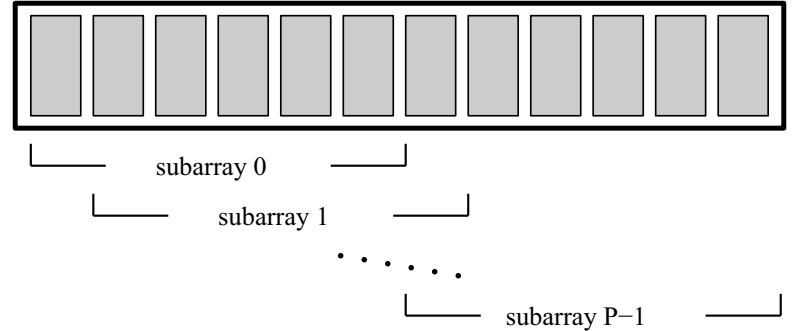

Fig. 2. Spatial smoothing. The array is divided into $P$ overlapping subarrays, and the covariance matrix is averaged across the array.

resulting covariance matrix is then given as the average over the subarrays

$$
\hat{\mathbf{R}}=\frac{1}{P} \sum_{p=0}^{P-1} \hat{\mathbf{R}}_{p}
$$

where $\hat{\mathbf{R}}_{p}$ is the $p$ th sub-covariance matrix estimate. Note that the spatial smoothing reduces the dimension of the covariance matrix to $L \times L$, and the dimension of the weight vector is reduced correspondingly. Thus, the number of degrees of freedom is reduced, which mean that the achievable resolution is also reduced.

For both the Rx and Tx apertures, diagonal loading is added to the covariance matrix. This introduces a robustness towards small disalignments. For more on robust adaptive beamforming methods see [12]. The diagonal loaded covariance matrix is given as

$$
\mathbf{R}=\hat{\mathbf{R}}+\epsilon \mathbf{I},
$$

where $\epsilon$ is small scalar value, and $\mathbf{I}$ is the $L \times L$ identity matrix.

\section{RESULtS}

Using Field II [13], [14], simulated SA data of 13 point targets has been obtained. The points are distributed at depths ranging from $40 \mathrm{~mm}$ to $70 \mathrm{~mm}$. For the simulations, a $5.5 \mathrm{MHz}, 64-$ element linear array transducer with $300 \mu \mathrm{m}$ pitch is used. The SA sequence consisting of 64 emissions is obtained using virtual sources with 11 active elements. For the MV beamforming, diagonal loading and a subarray size of $L=32$ are used both on the receiving and the transmitting apertures.

The proposed method ( $\mathrm{Rx}$ MV - Tx MV) is compared to two methods using data-independent apodization weighted on the transmitting aperture. These two methods are DAS using dynamic Hanning apodization weights with $f$-number of 2.5 on the receiving aperture and Boxcar weights on the transmitting aperture (Rx Hanning - Tx Boxcar), and MV on the receiving aperture and Boxcar weights on the transmitting aperture (Rx MV - Tx Boxcar).

The lateral variation of the three beamformed responses along two points placed laterally at a depth of $50 \mathrm{~mm}$ are shown in Fig. 3. It is seen that the MV beamformer provides significantly reduced main-lobe width compared to the conventional DAS beamformer. This has previously been demon-

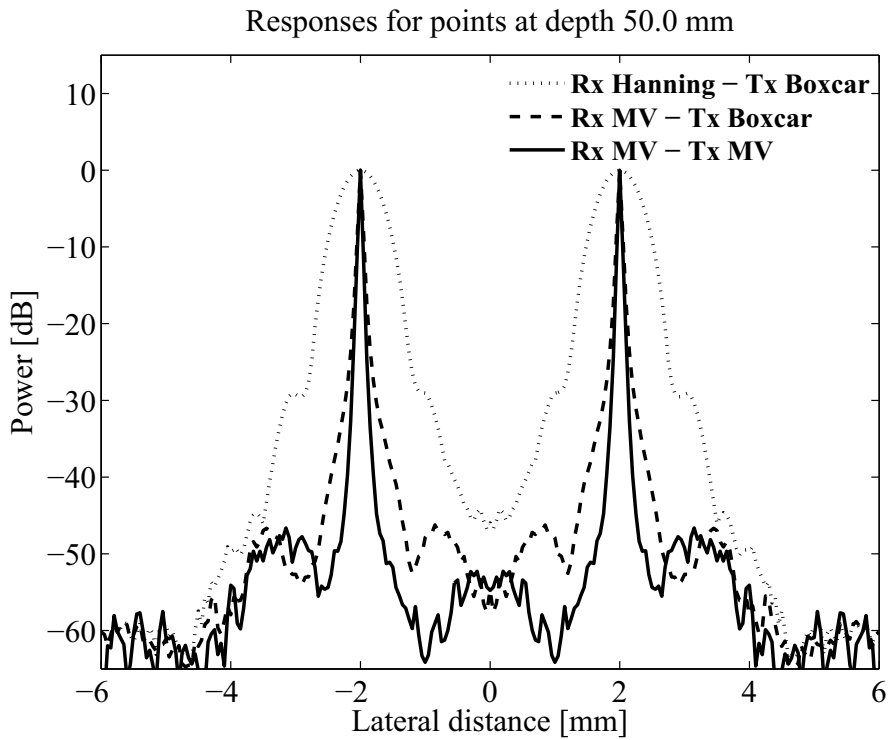

Fig. 3. Lateral variation of the beamformed responses in Fig. 4(a)-(c) along two points at a depth of $50 \mathrm{~mm}$.

strated in [5]. ${ }^{\mathrm{ii}}$ Furthermore, it is seen that the introduction of the data-dependent apodization weights on the transmitting aperture provides a reduction of the main-lobe width compared to these two methods.

For these two point targets placed laterally at a depth of $50 \mathrm{~mm}$, the three methods provide main-lobe widths (at $-30 \mathrm{~dB}$ ) of $\{2.20,0.47,0.26\} \mathrm{mm}$. Thus, the introduction of adaptive transmit apodization provides a reduction of the mainlobe width (at $-30 \mathrm{~dB}$ ) by a factor of 1.8 .

The three beamformed responses of the 13 point targets are shown in Fig. 4(a)-(c). It is seen that the main-lobe width has reduced significantly for the two adaptively beamformed responses in Fig. 4(b)-(c) compared to the data-independent DAS beamformed response in Fig. 4(a). Furthermore, it is observed that the adaptively beamformed response using adaptive apodization weights on both the receiving and the transmitting apertures in Fig. 4(c) provides a reduced mainlobe width. These observations are in consistency with Fig. 3 and with the fact that the number of degrees of freedom has increased significantly with the introduction of the datadependent apodization weights on the transmitting aperture.

\section{Discussion}

As mentioned previously, the introduction of data-dependent transmit apodization weights increases the number of degrees of freedom. Thus, the achievable resolution is potentially increased. These increments come at the expense of additional computational load. In the proposed method, it is suggested to carry out the two optimizations in two separate, subsequent steps. This introduces a matrix inversion for each step, and thus an increase of the computational load compared to the data-independent transmit apodization methods. Another

\footnotetext{
iiNote that in [5], the MV beamformer was implemented in the frequency domain. That provided an increased number of degrees of freedom compared to the implementation in the time domain, which is used in this paper.
} 


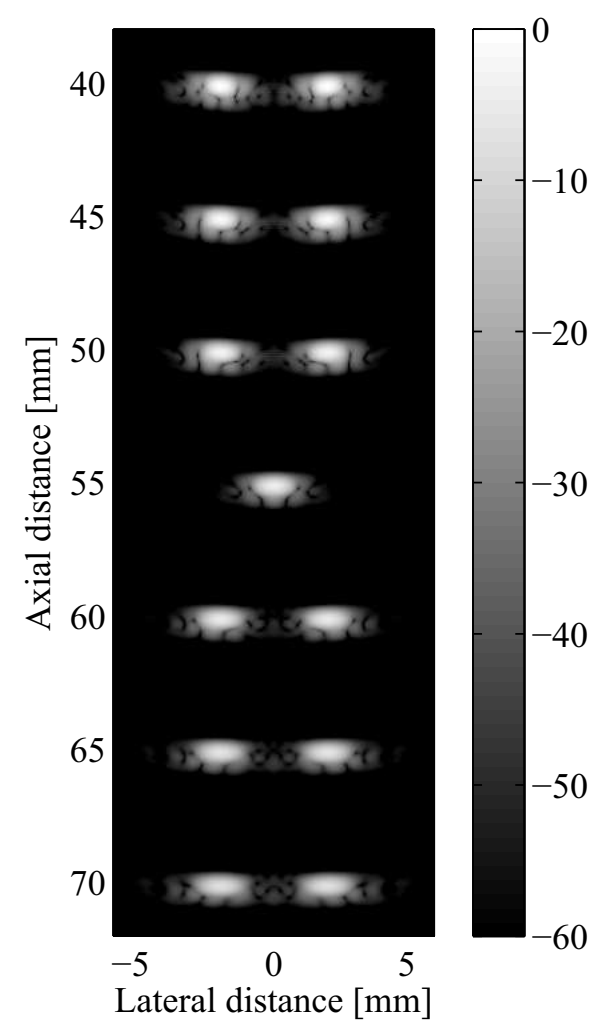

(a) Rx Hanning - Tx Boxcar

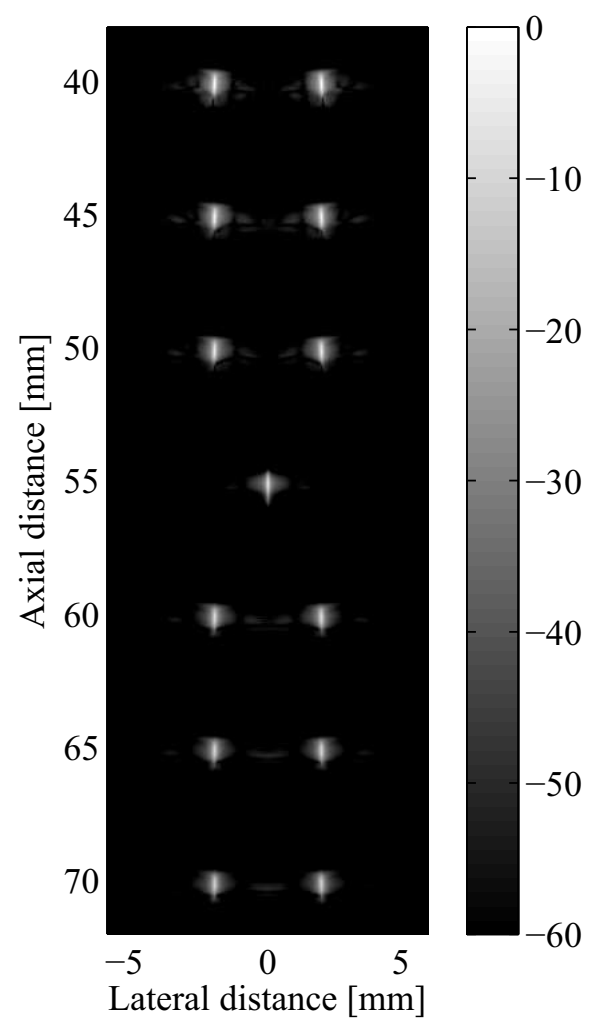

(b) Rx MV - Tx Boxcar

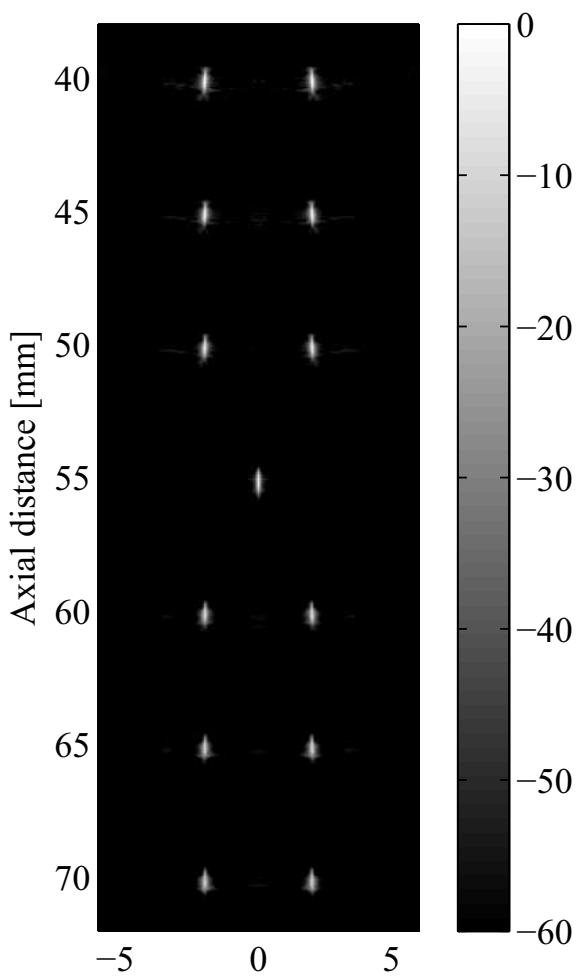

Lateral distance $[\mathrm{mm}]$

(c) Rx MV - Tx MV

Fig. 4. Beamformed responses for 13 discrete point targets. (a) Hanning apodization on the receiving and Boxcar on the transmitting aperture. (b) MV apodization on the receiving and Boxcar on the transmitting aperture. (c) MV apodization on both the receiving and transmitting apertures.

approach is to implement the optimization of the apodization weights on the receiving and transmitting apertures in one single optimization problem. However, that will introduce a significantly increased dimension of the covariance matrix. As the computational load of the matrix inversion is directly dependent on matrix dimension, it should be investigated further, whether or not the combination into one optimization problem will in fact reduce the computational load.

\section{Conclusions}

In this paper, a framework for applying adaptive, datadependent apodization weights on both the receiving and the transmitting aperture has been introduced. The suggested method has been validated using simulated synthetic aperture ultrasound data. The investigation has shown that the introduction of adaptive apodization weights on the transmitting aperture provides a main-lobe reduction (estimated at $-30 \mathrm{~dB}$ ) by a factor of 1.8 compared to the method using adaptive apodization weights on the receiving aperture only.

\section{ACKNOWLEDGMENT}

This work was supported by grant 26-04-0024 from the Danish Science Foundation and by B-K Medical ApS, Denmark.

\section{REFERENCES}

[1] J. A. Mann and W. F. Walker, "A Constrained Adaptive Beamformer for Medical Ultrasound: Initial Results," in Proc. IEEE Ultrason. Symp., Oct. 2002 , vol. 2, pp. $1807-1810$.
[2] M. Sasso and C. Cohen-Bacrie, "Medical Ultrasound Imaging Using the Fully Adaptive Beamformer," in Proc. IEEE Int. Conf. Acous., Speech, Sig. Pro., March 2005, vol. 2, pp. 489-492.

[3] F. Viola and W. F. Walker, "Adaptive Signal Processing in Medical Ultrasound Beamforming," in Proc. IEEE Ultrason. Symp., 2005, vol. 4, pp. 1980-1983.

[4] J.-F. Synnevåg, A. Austeng, and S. Holm, "Adaptive Beamforming Applied to Medical Ultrasound Imaging," IEEE Trans. Ultrason., Ferroelec., Freq. Contr., vol. 54, no. 8, pp. 1606-1613, Aug. 2007.

[5] I. K. Holfort, F. Gran, and J. A. Jensen, "Broadband Minimum Variance Beamforming for Medical Ultrasound Imaging," IEEE Trans. Ultrason., Ferroelec., Freq. Contr., vol. 56, no. 2, pp. 314-325, 2009.

[6] Z. Wang, J. Li, and R. Wu, "Time-Delay- and Time-Reversal-Based Robust Capon Beamformers for Ultrasound Imaging," IEEE Trans. Med. Imag., vol. 24, no. 10, pp. 1308-1322, 2005.

[7] F. Vignon and M. R. Burcher, "Capon Beamforming in Medical Ultrasound Imaging with Focused Beams," IEEE Trans. Ultrason., Ferroelec., Freq. Contr., vol. 50, no. 4, pp. 619-628, March 2008.

[8] S. I. Nikolov, Synthetic Aperture Tissue and Flow Ultrasound Imaging, Ph.D. thesis, Ørsted•DTU, Technical University of Denmark, 2800, Lyngby, Denmark, 2001.

[9] J. Capon, "High-Resolution Frequency-Wavenumber Spectrum Analysis," Proc. IEEE, vol. 57, no. 8, pp. 1408-1418, August 1969.

[10] J. S. Arora, Introduction to Optimum Design, McGraw-Hill, Inc., 1989.

[11] T.-J. Shan and T. Kailath, "Adaptive Beamforming for Coherent Signals and Interference," IEEE Trans. Acous., Speech, Sig. Pro., vol. 33, no. 3, pp. 527-536, June 1985.

[12] J. Li and P. Stoica, Robust Adaptive Beamforming, John Wiley \& Sons, New York, 2006

[13] J. A. Jensen and N. B. Svendsen, "Calculation of pressure fields from arbitrarily shaped, apodized, and excited ultrasound transducers," IEEE Trans. Ultrason., Ferroelec., Freq. Contr., vol. 39, pp. 262-267, 1992.

[14] J. A. Jensen, "Field: A program for simulating ultrasound systems," Med. Biol. Eng. Comp., vol. 10th Nordic-Baltic Conference on Biomedical Imaging, Vol. 4, Supplement 1, Part 1, pp. 351-353, 1996b. 